\title{
Livedoid vasculopathy - benefit of intravenous immunoglobulin in a refractory case
}

\author{
Stefan Cristian Dinescu', Andreea Lili Barbulescu'², Paulina Lucia Ciurea', \\ Roxana Mihaela Dumitrascu33, Beatrice Andreea Chisalau ${ }^{3}$, Cristina Dorina Parvanescu ${ }^{3}$, \\ Sineta Cristina Firulescu ${ }^{4}$, Florentin Ananu Vreju ${ }^{1}$ \\ ${ }^{1}$ Department of Rheumatology, University of Medicine and Pharmacy, Craiova, Romania \\ 2 Department of Pharmacology, University of Medicine and Pharmacy, Craiova, Romania \\ ${ }^{3}$ Doctoral School, University of Medicine and Pharmacy, Craiova, Romania \\ ${ }^{4}$ Department of Rheumatology, Emergency County Hospital, Craiova, Romania
}

\begin{abstract}
Livedoid vasculopathy is a rare vascular disease which typically manifests as recurrent ulcerative lesions on the lower extremities. It is classified as a vasculopathy, not a true vasculitis, and defined as a vasooclusive syndrome, caused by non-inflammatory thrombosis of the upper and mid-dermal venulae. Main disorders associated with LV include thrombophilias, autoimmune diseases and neoplasia. A triad of clinical features is present in most patients and consist of livedo racemosa (less frequently livedo reticularis), ulcerations and atrophie blanche. Management generally relies on antiplatelet drugs, anticoagulants, vasodilators and fibrinolytic therapy. Some benefit has been observed with intravenous immunoglobulin, colchicine, hyperbaric oxygen, while glucocorticoids are efficient to a lesser extent. This case report highlights a refractory clinical form with no identifiable predisposing condition, which proved responsive only to intravenous immunoglobulin.
\end{abstract}

Keywords: thrombosis, purpura, ulcer, intravenous immunoglobulins

\section{INTRODUCTION}

Livedoid vasculopathy (LV) is a rare vascular disease which typically manifests as recurrent ulcerative lesions on the lower extremities. Although the underlying pathogenesis is not well defined, most cases are linked to a procoagulant state. Associated conditions include inherited and acquired thrombophilias, cancer or autoimmune diseases. Relation to the latter and also the evidence of therapeutic response to immunosuppressive drugs supports the potential role of autoimmunity [1]. Onset of the disease usually occurs between 15 and 50 years with an estimated incidence of 1:100,000 and a female predominance of 3:1 [2]. The chronic course marked by recurrent exacerbations leads to porcelain white scars known as atrophie blanche. Numerous disease mimickers imply a particularly challenging differential diagnosis which usually relies on histpathological examination. This case report highlights an atypical and refractory clini- cal form with no identifiable predisposing condition, which proved responsive only to intravenous immunoglobulin.

\section{CASE PRESENTATION}

We report the case of a 41 year-old female patient with a long standing history of livedoid vasculopathy of approximately 10 years. Although no relevant disease history was reported before her diagnosis, her family history was significant, with autoimmunity on her mother's side in the form of autoimmune thyroiditis and autoimmune hepatitis, while her father had been diagnosed with Hodgkin lymphoma.

At onset, the disease manifested with features of livedo reticularis, purpuric macules and papules located on the lower leg, around the ankle and on the dorsa of the foot. Cutaneous lesions were symmetrical, accompanied by slight edema. After 5 months, skin ulcers started to emerge, that caused some pain 

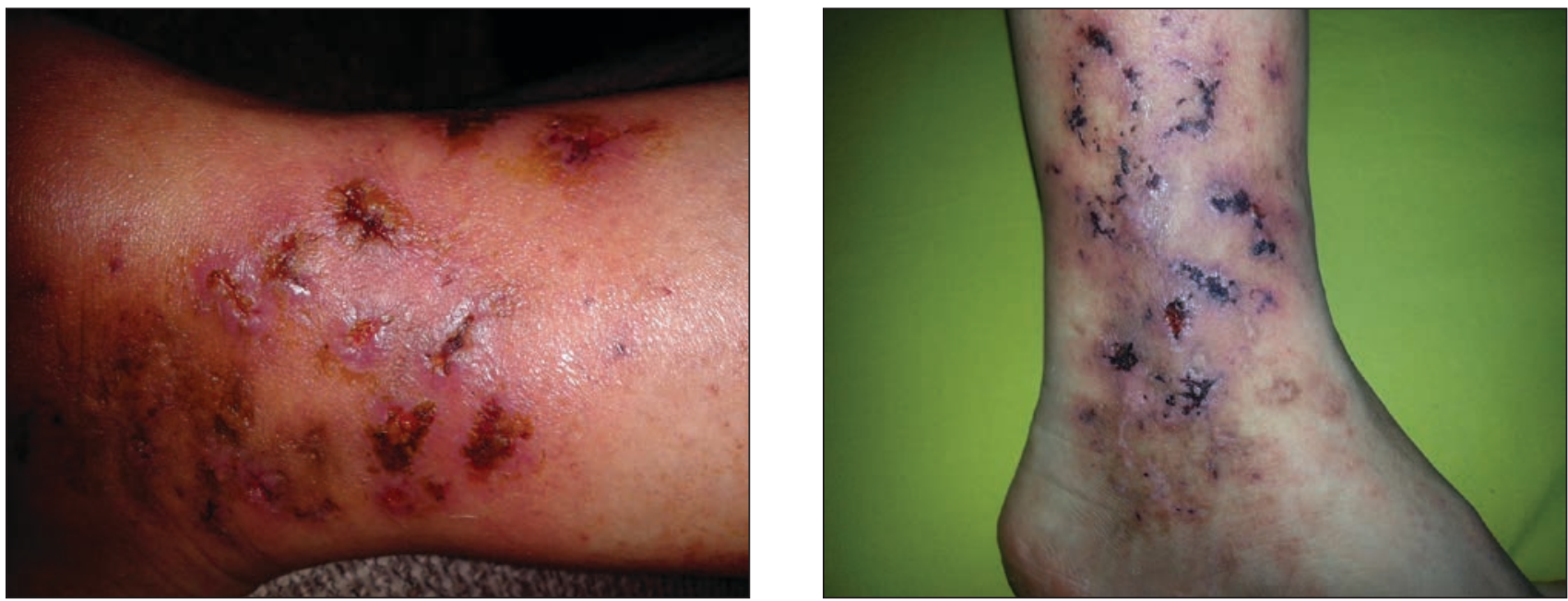

FIGURE 1. (A) cutaneous lesions 1 year after disease onset; note the presence of multiple ulcerations with perilesional inflammation, located around the ankle, some of which have merged to form larger reticulated and slow healing ulcers;

(B) ulcer formations covered by hematic crusts persist 1 year into the treatment with corticosteroid, anti-platelet drugs and vasodilators

and had a major aesthetic impact (Figure 1A). Disease course was marked by recurrent exacerbations with new ulcers developing, while scarring in resolved lesions lead to the typical atrophie blanche features.

Initial investigations took into account the family background for autoimmunity and the clinical features of purpuric rash, which raised the suspicion of a systemic vasculitis. Antinuclear antibody panel test, along with antineutrophil cytoplasmic antibodies were negative. Development of necrotic ulcers was also considered as result of a prothrombotic condition. Screening for inherited and acquired thrombophilia was negative and included: protein $\mathrm{C}$ and $\mathrm{S}$ deficiency, prothrombin mutation and factor V Leiden, antithrombin III deficiency, cryoglobulinemia and homocysteinemia. Antiphospholipid syndrome was also excluded. The only serum abnormality which could hint to a possible autoimmune disease was a constant $\mathrm{C} 3$ hypocomplementemia.

Skin biopsy was necessary at this point given the scarcity of serum markers and also the diagnostic ambiguity based solely on clinical features. Histopathological examination of the skin sample showed multiple thrombi formation in dermal vessels, with fibrin deposits inside the lumen and vessel wall. Few necrotic areas were present in the epidermis. More importantly, perivascular inflammation and leukocytoclasia were absent. As a result, a diagnosis of livedoid vasculopathy could be established.

Initial therapy targeted the vascular insufficiency using pentoxifilin, soludexide and diosminum. After diagnosis of LV was confirmed, oral corticosteroids and hidroxicloroquine were added, but could not provide significant improvement (Figure 1B). Over the next five years, the patient went through various treatment trials which included aspirin, colchicine, cilostazol and dapsone.

With no apparent benefit from the aforementioned therapies and poor healing of ulcerative lesions, a new approach was considered using intravenous immunoglobulin (IVIG) administered once per month. Currently, the patient has completed a total of nine cycles of IVIG, which were divided into a 3-month course and a 6-month course during the past 2 years. A significant improvement of skin lesions was obtained after the first IVIG course, with persistent healing and absence of new ulcer formation throughout the second course (Figure 2).

Maintenance therapy includes: dapsone, diosminum, soludexide, aspirin and colchicine. Follow-up investigations provided no additional information regarding a prothrombotic state, malignancy or autoimmunity, except a constant $\mathrm{C} 3$ hypocomplementemia. The patient never developed other features suggestive of a connective tissue disease.

\section{DISCUSSIONS}

Livedoid vasculopathy remains an unusual diagnosis due to its rare occurrence and lack of familiarity among practitioners. The earliest report by Millman in 1929 [1], described this entity as atrophie blanche. The actual term of "livedoid vasculopathy" was first introduced by Bard and Winkelmann [3]. Other terms used to designate LV include: livedo/livedoid vasculitis, segmental hyalinizing vasculitis, livedo reticu- 

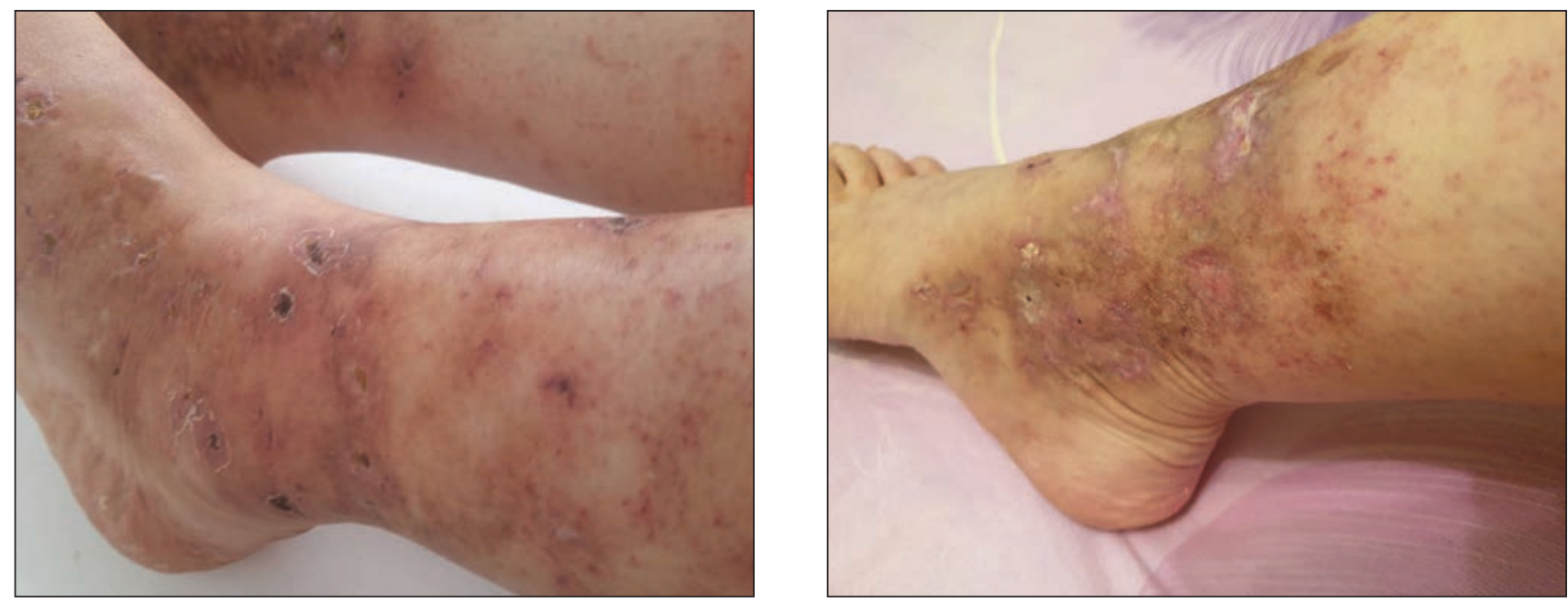

FIGURE 2. (A) clinical outcome after first 3 cycles of IVIG; note the significant improvement in number and size of ulcers; (B) classic porcelain-white atrophic scarring and residual hyperpigmentation, with no new ulcer formations, throughout the second course of IVIG

laris with summer ulcerations or PURPLE (painful purpuric ulcers with reticular pattern on the lower extremities) [4].

Current knowledge on pathogenesis and evidence of specific histopathology features helped to distinguish it from vasculitis, as it was considered in earlier reports. LV is defined as a vasooclusive syndrome, caused by non-inflammatory thrombosis of the upper and mid-dermal venulae [5]. Given its heterogeneous ethiopathogenic background, it should be viewed as a cutaneous manifestation of a prothrombotic disease. Main disorders associated with LV include thrombophilias, autoimmune diseases and neoplasia [1]. However, up to $20 \%$ of cases remain idiopathic [5]. This was also the case for our patient, in which all initial and subsequent tests could not link the LV diagnosis to predisposing disease state.

The underlying prothrombotic mechanism depends on the predisposing condition and falls into one of the three categories of Virchow's triad: endothelial damage, stasis of the blood and hypercoagulability state [4]. Diseases associated with endothelial damage include: systemic lupus erythematous (SLE), systemic scleroderma, mixed connective tissue disease, rheumatoid arthritis, polyarteritis nodosa and hyperhomocysteinemia [4,6]. Hyperviscosity syndrome can be linked to cryoglobulinemia, heavy chain disease or chronic myelogenous leukemia [4]. Hypercoagulability related to thrombophilia is a possible cause of LV and requires screening for coagulation factor deficiencies (protein $\mathrm{C}, \mathrm{S}$, antithrombin) or mutations (factor V Leiden and prothrombin). LV can be found in conjunction with antiphospholipid antibodies. Some authors have considered LV also as a potential manifestation of seronegative antiphospholipid syndrome in SLE patients with recurrent thrombosis [7].

A triad of clinical features is present in most patients and consist of livedo racemosa (less frequently livedo reticularis), ulcerations and atrophie blanche. In initial phases, reduced blood flow results in erythematous-violaceous streaks with net-like pattern. Purpuric macules and papules develop symmetrically on the lower limbs, and can be accompanied by pain and pruritus [1]. These evolve into hemorrhagic vesicles which become small ulcers when ruptured and tend to merge in larger reticulated ulcerations. After a period of several months, the lesions slowly heal and turn into irreversible white porcelain scars called atrophie blanche or capilaritis alba [2]. The scars may feature peripheral telangiectasia. Lesions in different stages of evolution can coexist. LV does not develop systemic involvement; this is rather related to an associated autoimmune disease. Mononeuritis multiplex caused by vasa nervorum thrombosis may lead to paresthesia or hyperesthesia in some patients [4]. LV has a chronic course with recurrent exacerbations.

Accurate diagnosis has a 5-year delay on average which prevents early treatment in many patients [2]. Misdiagnosis leads to irreversible skin lesions and psychological scarring. A histopathology examination is essential, taking into account the wide differential diagnosis. Adequate skin sample should be obtained from erythematous-purpuric lesions, while avoiding ulcerated areas [4]. Characteristic histopathological features are fibrin deposits in the vascu- 
lar lumen of upper and mid-dermal capillaries, segmental hyalinization of vessel wall, and lack of leukocytoclasia. Based on histopathology, LV can be clearly differentiated from an immune-mediated necrotizing vasculitis. Perivascular infiltration is absent or minimal and predominantly lymphocytic. Neutrophils are found around ulcerated areas. Tissue samples must include the dermo-hypodermal junction. This is important for the differentiation from polyarteritis nodosa which can imitate LV clinically, but develops vessel occlusion in the deep dermis. Other, features of LV include: hyalinized fibrin rings, epidermis necrosis due to ischemia and extravasation of red blood cells in the superficial dermis which leads to cutaneous hemosiderosis [5]. Hyalinization of the dermis, epidermis atrophy and teleangiectazia of upper dermis are changes seen in late stages. Changes seen in direct immunofluorescence do not provide any specific information in regard to diagnosis; these include deposition of fibrin, immunoglobulin, and complement [4].

Management of LV patients is non-standardized, dependent on personal experience and evidence from case reports and case series. Main goals of therapy are improvement of skin lesions, pain relief and prevention of recurrence. Recommendations include smoking cessation, compression stockings, use of anticoagulants and antiplatelet drugs, anabolic steroids, vasodilators, tissue plasminogen activator, psoralens plus ultraviolet A (PUVA), IVIG or hyperbaric oxygen [6]. Micieli et al. published in a systematic review treatment data from $339 \mathrm{LV}$ patients [8]. Anticoagulants showed high efficiency and were the Conflict of interest: none declared Financial support: none declared

\section{REFERENCES}

1. Vasudevan B, Neema S, Verma R. Livedoid vasculopathy: A review of pathogenesis and principles of management. Indian J Dermatol Venereol Leprol. 2016;82:478-488.

2. Kerk N, Goerge T. Livedoid vasculopathy - a thrombotic disease. Vasa. 2013;42(5):317-322.

3. Bard JW, Winkelmann RK. Livedo vasculitis. Segmental hyalinizing vasculitis of the dermis. Arch Dermatol. 1967;96:489-99.

4. Criado PR, Rivitti EA, Sotto MN, et al. Livedoid vasculopathy: an intringuing cutaneous disease. An Bras Dermatol. 2011;86(5):961977

5. Freitas TQ, Halpern I, Criado PR. Livedoid vasculopathy: a compelling diagnosis. Autops Case Rep. 2018;8(3):e2018034.

6. Gonzalez-Santiago TM, Davis MD. Update of management of connective tissue diseases: livedoid vasculopathy. Dermatol Ther. 2012;25(2):183-94. most commonly used class in monotherapy. Antiplatelet drugs found effective in LV treatment include: aspirin, dipyridamole and pentoxiphylline. Anabolic steroids such as danazol proved beneficial through the interference with hepatic production of coagulation factors. By contrast, glucocorticoids have low success rates and this is due to the pauci-immune nature of this disease [5]. Treatment with IVIG has provided encouraging results in individual cases and some large group studies. Kreuter et al. studied low dose IVIG $(0.5 \mathrm{~g} / \mathrm{kg})$ in 9 patients and obtained significant regression of skin lesions in each case [9]. In a study by Monshi et al. on 11 patients treated with $2 \mathrm{~g} / \mathrm{kg}$ IVIG, complete remission was obtained in some cases after only 3 cycles, although the vast majority of flares (89\%) required 6 cycles [10]. In our experience, significant improvement was observed after first 3 cycles, while persistent remission was obtained following the second 6 cycle course.

\section{CONCLUSIONS}

Livedoid vasculopathy is a chronic cutaneous manifestation due to microvascular thrombosis, which can develop in the setting of various connective tissue diseases. Rheumatologists need to be aware of this entity that is often misdiagnosed and becomes challenging when appropriate treatment is delayed. Slow healing ulcerations with periodic exacerbations and development of irreversible scars significantly impacts patient's quality of life. There is a need for high quality evidence from control studies regarding optimal treatment.
7. Sopeña B, Pérez-Rodríguez MT, Rivera A, Ortiz-Rey JA, Lamas J, Freire-Dapena MC. Livedoid vasculopathy and recurrent thrombosis in a patient with lupus: seronegative antiphospholipid syndrome? Lupus. 2010;19(11):1340-3.

8. Micieli R, Alavi A. Treatment for Livedoid Vasculopathy: A Systematic Review. JAMA Dermatol. 2018;154(2):193-202.

9. Kreuter A, Gambichler T, Breuckmann F, Bechara FG, Rotterdam S, Stücker M, Altmeyer P. Pulsed intravenous immunoglobulin therapy in livedoid vasculitis: an open trial evaluating 9 consecutive patients. J Am Acad Dermatol. 2004;51(4):574-9.

10. Monshi B, Posch C, Vujic I, Sesti A, Sobotka S, Rappersberger K. Efficacy of intravenous immunoglobulins in livedoid vasculopathy: long-term follow-up of 11 patients. J Am Acad Dermatol. 2014;71(4):738-44. 